\title{
The Evidential Conditional
}

\section{Vincenzo Crupi $^{1}$ (D) Andrea lacona ${ }^{1}$}

Received: 5 December 2019 / Accepted: 5 October 2020 / Published online: 31 December 2020

(c) The Author(s) 2020

\begin{abstract}
This paper outlines an account of conditionals, the evidential account, which rests on the idea that a conditional is true just in case its antecedent supports its consequent. As we will show, the evidential account exhibits some distinctive logical features that deserve careful consideration. On the one hand, it departs from the material reading of 'if then' exactly in the way we would like it to depart from that reading. On the other, it significantly differs from the non-material accounts which hinge on the Ramsey Test, advocated by Adams, Stalnaker, Lewis, and others.
\end{abstract}

\section{Overview}

Logicians have always been tempted by the thought that 'if then' expresses a relation of support, which can be articulated by saying that the antecedent of a conditional must provide a reason to accept its consequent, or that the inference from its antecedent to its consequent must be justified. According to a view that is traditionally attributed to the Stoics, and most prominently to Chrysippus, conditionals are inherently related to arguments, in that an argument is valid just in case the conditional formed with the conjunction of its premises as antecedent and its conclusion as consequent is true. ${ }^{1}$ The same sort of link between conditionals and arguments has been postulated by several authors in the subsequent debates on conditionals. It is no surprise that the term 'consequent', which derives from the latin 'consequens', recalls to mind the notion of consequence. ${ }^{2}$ Here is a telling quote from Mill:

\footnotetext{
1 Sextus Empiricus, Outlines of Pyrrhonism II, 137.

2 Peter Abelard, and other medieval logicians after him, used the term 'consequentia' to refer to a conditional. As the word suggests, the underlying idea was that conditionals are characterized by some consequence relation, see Kneale and Kneale (1962), p. 215.
}

Vincenzo Crupi

vincenzo.crupi@unito.it

Andrea Iacona

andrea.iacona@unito.it

1 Center for Logic, Language, and Cognition, Department of Philosophy and Education, University of Turin, Turin, Italy 
When we say, If the Koran comes from God, Mohammed is the prophet of God, we do not intend to affirm either that the Koran does come from God, or that Mohammed is really his prophet. Neither of these simple propositions may be true, and yet the truth of the hypothetical proposition may be indisputable. What is asserted is not the truth of either of the propositions, but the inferribility of the one from the other. ${ }^{3}$

The thought that 'if then' expresses a relation of support owes its intuitive appeal to the fact that in most cases it is quite natural to paraphrase conditionals by using words such as 'reason' or 'infer'. Consider the following examples:

(1) If it's pure cashmere, it will not shrink

(2) If you drink a beer, you'll feel better

(3) If it is snowing, then it is cold

It seems correct to say that the antecedent of (1) supports its consequent, as the following reformulations suggest:

(4) If it's pure cashmere, that is a reason for thinking that it will not shrink

(5) If it's pure cashmere, we can infer that it will not shrink

Similar considerations hold for (2) and (3). What one wants to say when one utters (2) is that drinking a beer makes you feel better, so that if you drink it, you'll experience that effect. In the case of (3), again, the antecedent provides a reason to accept the consequent, although here the event described by the antecedent does not cause the event described by the consequent.

Of course, there are cases in which no paraphrase in terms of 'reason' or 'infer' is available. Typically, concessive conditionals do not admit reformulations along the lines suggested. Suppose we intend to go out for a walk and we hope that it will be sunny. We can nevertheless assert what follows:

(6) If it rains, we will go

In this case it would be inappropriate to say that the rain provides evidence to think that we will go. What we mean, instead, is that we will go anyway, that is, in spite of the rain. So, (7) and (8) seem correct reformulations of (6):

(7) Even if it rains, we will go

(8) If it rains, we will still go

More generally, concessive conditionals are suitably phrased by using 'even if' or 'still', and do not imply support in the sense considered. Nonetheless, the range of

${ }^{3}$ Mill (1843), p. 102. 
cases in which the notion of support seems pertinent is sufficiently large and representative to deserve separate study. ${ }^{4}$

Despite the plain intelligibility of paraphrases such as (4) and (5), the notion of support proves hard to capture at the formal level. This explains the multiplicity and the heterogeneity of the attempts that have been made so far to define a connective with the property desired. At least two main lines of thought have been pursued. One option is to treat conditionals as strict conditionals and define support in terms of some sort of necessitation: a conditional is true just in case it is impossible that its antecedent is true and its consequent is false. ${ }^{5}$ Another option is to develop a nonmonotonic theory of conditionals which aims at capturing the intuitive understanding of support. ${ }^{6}$

The account outlined here belongs to the second category. The interpretation of 'if then' that we will explore develops the idea that a conditional is true just in case its antecedent provides evidence for its consequent, where 'provides evidence for' is another way of saying 'supports'. This interpretation may be regarded as one coherent reading of 'if then', although it is not necessarily the only admissible reading. We will not address the thorny question whether there is a unique correct analysis of 'if then', because the main points that we will make can be acknowledged without assuming an affirmative answer to that question. If different readings of 'if then' are equally admissible, the evidential interpretation is one of them.

Interestingly, the notion of support seems to apply equally well to indicative conditionals and to counterfactuals. Although we will focus on indicative conditionals, what we will say about this notion can easily be extended to counterfactuals. In particular, the distinction between evidential and concessive readings of 'if then' is orthogonal to the distinction between indicative and subjunctive conditionals. For example, the following sentences exhibit the same difference that obtains between (1) and (6):

(9) If it were cashmere, it would not shrink

(10) If it were raining, we would go

\footnotetext{
${ }^{4}$ Gomes (2019) aptly emphasizes the contrast between reformulations such as (4) and (5) and reformulations such as (7) and (8), and draws a distinction between "implicative" and concessive conditionals. Some linguists use the label "inferential" to refer to a specific category of conditionals, see for instance Dancygier (1998), Declerck and Reed (2001), Dancygier and Sweetser (2005). Independently of such classifications, all that matters for our purposes is that many conditionals are implicative or inferential in the obvious sense that they reflect or express some kind of reasoning.

5 This option has been developed in different ways by Lycan (2001), Gillies (2009), Kratzer (2012), Iacona (2018), and others.

6 Among the most recent attempts, Rott (1986) contains a pioneering discussion of 'if' and 'because', relying on a variation of the belief revision formalism. The ranking-theoretic account offered in Spohn (2013) explicitly involves the idea of the antecedent as providing a reason for the consequent. The approach to conditionals outlined in Douven $(2008,2016)$ employs the notion of evidential support from Bayesian epistemology. Wenmackers et al. (2013), van Rooij and Schulz (2019), and Berto and Özgün (2020) provide further examples.
} 
While (9) can be paraphrased by means of sentences analogous to (4) and (5), the most appropriate reformulations of (10) are sentences analogous to (7) and (8).

The structure of the paper is as follows. Section 2 provides a first informal sketch of the evidential account and introduces the symbol $\triangleright$, which represents our reading of 'if then'. ${ }^{7}$ Section 3 defines a language that includes $\triangleright$ in addition to the standard symbols of modal propositional logic. Sections 4-8 spell out some important logical properties of $\triangleright$. Section 9 compares the evidential account with other accounts, in particular with those suggested by Adams, Stalnaker, and Lewis. Finally, Sect. 10 considers a probabilistic version of the analysis suggested, which defines assertibility in terms of a quantitative measure of support.

\section{The Core Idea}

A rather solid intuition about conditionals such as (1)-(3) is that there seems to be some sort of clash between their antecedent and the negation of their consequent. For example, insofar as one accepts (1), one will find that 'it's pure cashmere', and 'it will shrink' do not go well together, where 'it will shrink' amounts to the negation of 'it will not shrink'. Similar considerations hold for (2) and (3). Long time ago, Chrysippus expressed this intuition in a remarkably straightforward way:

A conditional holds whenever the denial of its consequent is incompatible with its antecedent. ${ }^{8}$

Assuming that the negation of a sentence is true just in case the sentence is false, this is to say that a conditional holds whenever the falsity of its consequent is incompatible with the truth of its antecedent. Of course, the word 'incompatible' may be construed in different ways, and nobody knows what Chrysippus exactly had in mind. But we believe that there is at least one coherent reading of his claim that accords with the thought that 'if then' epxresses a relation of support. ${ }^{9}$

The core idea of the evidential account is that a conditional $\alpha \triangleright \beta$ is true if and only if $\alpha$ and $\sim \beta$ are incompatible in the following sense: if $\alpha$ is true, then $\beta$ cannot easily be false, and if $\beta$ is false, then $\alpha$ cannot easily be true. This is a conjunction because we assume that incompatibility is a symmetric relation: $\alpha$ is incompatible with $\sim \beta$ if and only if $\sim \beta$ is incompatible with $\alpha$. So, the incompatibility claim can be read both from left to right and from right to left. The first condition-if $\alpha$ is true, then $\beta$ cannot easily be false-expresses the left-to-right reading of the claim, that is, ' $\alpha$ is incompatible with $\sim \beta$ '. The second condition-if $\beta$ is false, then $\alpha$ cannot easily be true-expresses the right-to-left reading of the claim, which emerges clearly in Chrysippus' formulation, that is, ' $\sim \beta$ is incompatible with $\alpha$ '.

These two conditions can be spelled out in terms of comparative measures of distance from the actual world. The first requires that the worlds in which $\alpha$ is true and

\footnotetext{
7 This symbol is borrowed from Spohn (2013).

8 Sextus Empiricus, Outlines of Pyrrhonism, II, 110-112.

${ }^{9}$ For extensive discussions of the passage quoted, see Sanford (2003), p. 25, Lenzen (2019a), pp. 15-19.
} 
$\beta$ is false are distant from the actual world if compared with those in which $\alpha$ and $\beta$ are both true. This is essentially the Ramsey Test as understood by Stalnaker and Lewis: in the closest worlds in which $\alpha$ is true, $\beta$ must be true as well. ${ }^{10}$ The second, which is seldom discussed in the literature on conditionals, requires that the worlds in which $\alpha$ is true and $\beta$ is false are distant from the actual world if compared with those in which $\alpha$ and $\beta$ are both false. This may be labeled Reverse Ramsey Test: in the closest worlds in which $\beta$ is false, $\alpha$ must be false as well. We will call Chrysippus Test the conjunction of the Ramsey Test and the Reverse Ramsey Test, as we take the quote above to suggest that the latter is at least as important as the former.

To illustrate the Chrysippus Test, consider the following diagrams, where 11, $10,01,00$ indicate the combinations of truth values of $\alpha$ and $\beta$, and the length of each dashed line indicates the distance from the actual world of the closest world in which the respective combination occurs:

$$
\begin{aligned}
& ---11 \\
& 01---10 \\
& --00 \\
& -----11 \\
& 01-10 \\
& -00 \\
& --11 \\
& ---10 \\
& ----100
\end{aligned}
$$

In the first case $\alpha \triangleright \beta$ is true, because both the Ramsey Test and the Reverse Ramsey Test are satisfied: 11-worlds and 00-worlds are closer than 10-worlds. In the second case $\alpha \triangleright \beta$ is false, because it does not pass the Ramsey Test: 10 -worlds are closer than 11-worlds. In the third case, again, $\alpha \triangleright \beta$ is false, but this time because it does not pass the Reverse Ramsey Test: 10-worlds are closer than 00-worlds.

The Chrysippus Test is stronger than the Ramsey Test exactly in the way that seems required in order to preserve the intuition that $\alpha$ must support $\beta$. Consider a situation like that depicted in the third diagram, that is, a scenario in which $\beta$ is very likely regardless of $\alpha$, and in which we would be inclined to say that $\beta$ does not hold in virtue of $\alpha$. For example,

(11) If you drink a beer, the sun will rise tomorrow

10 The Ramsey Test comes from Ramsey (1929). Stalnaker (1991) and Lewis (1973) adopt the modal interpretation suggested. 
In this case the Reverse Ramsey Test, unlike the Ramsey Test, is not satisfied. Although the closest worlds in which you drink a beer are worlds in which the sun will rise tomorrow, it is not the case that the closest worlds in which the sun will not rise tomorrow are worlds in which you don't drink a beer. Even if the absence of sunrise is a remote possibility, its distance from the actual world does not depend on your beer. Thus, an account of conditionals based on the Chrysippus Test, unlike one which relies solely on the Ramsey Test, will predict that (11) is false. The latter prediction is exactly what one should expect from the evidential interpretation: the antecedent of (11) does not provide a reason for accepting its consequent. ${ }^{11}$

In order to provide a perspicuous representation of comparative measures of distance, we will employ the system of spheres adopted by Lewis in his semantics for counterfactuals. We will imagine non-actual worlds as ordered in a set of spheres around the actual world, depending on their degree of similarity to the actual world. This is a reasonably neutral formal tool which can be used without being committed to the rest of Lewis' view about conditionals and modal metaphysics. ${ }^{12}$

In the framework of the system of spheres, the Ramsey Test requires that, unless $\alpha$ is impossible, that is, true in no world, there is a 10-free sphere-a sphere that contains no 10 -worlds - in which $\alpha$ is true in some world. For if there is such a sphere, some 11-worlds are closer to the actual world than any 10-world. The Reverse Ramsey Test requires instead that, unless $\beta$ is necessary, that is, true in every world, there is a 10 -free sphere in which $\beta$ is false in some worlds. For if there is such a sphere, some 00-worlds are closer to the actual world than any 10-world. The Chrysippus Test thus requires that, unless $\alpha$ is impossible or $\beta$ is necessary, there is a 10 -free sphere in which $\alpha$ is true in some world and $\beta$ is false in some world. $\alpha \triangleright \beta$ is nonvacuously true when there is such a sphere.

This account of non-vacuous truth can reasonably be combined with a standard characterization of vacuous truth. We will assume that $\alpha \triangleright \beta$ is vacuously true when $\alpha$ is impossible, for it is vacuously the case both that if $\alpha$ is true, then $\beta$ cannot easily be false, and that if $\beta$ is false, then $\alpha$ cannot easily be true. Similarly, we will assume that $\alpha \triangleright \beta$ is vacuously true when $\beta$ is necessary, for again both conditions are satisfied. In other words, if $\alpha$ is impossible or $\beta$ is necessary, then $\alpha$ and $\sim \beta$ are vacuously incompatible.

\section{Definitions}

To phrase in formal terms what we have just said, we will define a language called $\mathrm{L}$. The symbols of $\mathrm{L}$ are the letters $p, q, r, \ldots$, the connectives $\sim, \supset, \wedge, \vee, \triangleright, \square, \diamond$, and the brackets (, ). The formulas of $L$ are defined by induction in the usual way: $p, q, r \ldots$ are formulas; if $\alpha$ is a formula, $\sim \alpha, \square \alpha, \diamond \alpha$ are formulas; if $\alpha$ and $\beta$ are formulas, $\alpha \supset \beta, \alpha \wedge \beta, \alpha \vee \beta, \alpha \triangleright \beta$ are formulas.

\footnotetext{
11 Rott (1986), Douven (2008), and others have discussed similar examples and explained their unacceptability in different ways.

12 Lewis (1973), pp. 13-19.
} 
Definition 1 Given a non-empty set $W$, a system of spheres $O$ over $W$ is an assignment to each $w \in W$ of a set $O_{w}$ of non-empty sets of elements of $W$-a set of spheres around $w$-such that:

1. if $S \in O_{w}$ and $S^{\prime} \in O_{w}$, then either $S \subseteq S^{\prime}$ or $S^{\prime} \subseteq S$;

2. $\{w\} \in O_{w}$;

3. if $S \neq \bigcup O_{w}$, then there is a $S^{\prime}$ such that $S \subset S^{\prime}$ and $S^{\prime} \subseteq S^{\prime \prime}$ for every $S^{\prime \prime}$ such that $S \subset S^{\prime \prime}$.

Clause 1 says that $O_{w}$ is nested. This condition rules out that two spheres $S, S^{\prime}$ and two worlds $w^{\prime}, w^{\prime \prime}$ are such that $w^{\prime} \in S$ but $w^{\prime} \notin S^{\prime}$ and $w^{\prime \prime} \in S^{\prime}$ but $w^{\prime \prime} \notin S$. For it cannot be the case that $w^{\prime}$ is more similar to $w$ than $w^{\prime \prime}$ and $w^{\prime \prime}$ is more similar to $w$ than $w^{\prime}$.

Clause 2 implies that $O_{w}$ is centered on $w$. If $\{w\} \in O_{w}$, then by clause 1 we have that, for every $S \in O_{w},\{w\} \subseteq S$, given that $S$ is assumed to be non-empty. This means that $w$ belongs to every sphere around $w$. The idea is that the innermost sphere is a singleton because no other world is as similar to $w$ as $w$ itself is.

Clause 3 states the limit assumption, according to which, for every sphere smaller than $\bigcup O_{w}$, there is a smallest sphere around $S$ : getting closer and closer to $S$ we eventually reach a limit. In the specific case in which $S=\{w\}$, this means that some sphere contains the worlds closest to $w$. Although Lewis finds this assumption questionable for metaphysical reasons, we think that we can live with it. ${ }^{13}$

Definition 2 A model for $L$ is an ordered triple $\langle W, O, V\rangle$, where $W$ is a nonempty set, $O$ is a system of spheres over $W$, and $V$ is a valuation function such that, for each atomic formula $\alpha$ of $L$ and each $w \in W, V(\alpha, w) \in\{1,0\}$.

Definition 3 The truth conditions of a formula of $L$ in a world $w$ in a model are as follows:

1. If $\alpha$ is atomic, $[\alpha]_{w}=1$ iff $V(\alpha, w)=1$;

2. $[\sim \alpha]_{w}=1$ iff $[\alpha]_{w}=0$;

3. $[\alpha \wedge \beta]_{w}=1$ iff $[\alpha]_{w}=1$ and $[\beta]_{w}=1$;

4. $[\alpha \vee \beta]_{w}=1$ iff either $[\alpha]_{w}=1$ or $[\beta]_{w}=1$;

5. $[\alpha \supset \beta]_{w}=1$ iff either $[\alpha]_{w}=0$ or $[\beta]_{w}=1$;

6. $[\alpha \triangleright \beta]_{w}=1$ iff the following conditions hold:

(a) for every $w^{\prime} \in \bigcup O_{w}$, if $[\alpha]_{w^{\prime}}=1$ and there are no $w^{\prime \prime}$ and $S$ such that $w^{\prime \prime} \in S, w^{\prime} \notin S$, and $[\alpha]_{w^{\prime \prime}}=1$, then $[\beta]_{w^{\prime}}=1$;

\footnotetext{
${ }^{13}$ Further constraints on $O$ might be added. One is closure under union: if $S \subseteq O_{w}$ and $\bigcup S$ is the set of all $w^{\prime}$ such that $w^{\prime}$ belongs to some member of $S$, then $\bigcup S \in O_{w}$. Another is closure under intersection: if $S \subseteq O_{w}$ and $\bigcap S$ is the set of all $w^{\prime}$ such that $w^{\prime}$ belongs to every member of $S, \bigcap S \in O_{w}$. A third constraint is uniformity: for every $w, w^{\prime} \in W, \bigcup O_{w}=\bigcup O_{w^{\prime}} ;$. Although each of these additional constraint is reasonable, none of them is strictly necessary for our purposes. See Lewis (1973), pp. 14-15, 120-121.
} 
(b) for every $w^{\prime} \in \bigcup O_{w}$, if $[\beta]_{w^{\prime}}=0$ and there are no $w^{\prime \prime}$ and $S$ such that $w^{\prime \prime} \in S, w^{\prime} \notin S$, and $[\beta]_{w^{\prime \prime}}=0$, then $[\alpha]_{w^{\prime}}=0$;

$7 \quad[\square \alpha]_{w}=1$ iff, for every $w^{\prime}$ in every $S \in O_{w},[\alpha]_{w^{\prime}}=1$;

$8[\diamond \alpha]_{w}=1$ iff, for some $w^{\prime}$ in some $S \in O_{w},[\alpha]_{w^{\prime}}=1$.

In clause 6, (a) expresses the Ramsey Test: $\beta$ must be true in the closest worlds in which $\alpha$ is true. (b) expresses the Reverse Ramsey Test: $\alpha$ must be false in the closest worlds in which $\beta$ is false. Note that if $\alpha$ is impossible, the antecedent of (a) is false for every world, and the consequent of (b) is true for every world. Similarly, if $\beta$ is necessary, the consequent of (a) is true for every world, and the antecedent of (b) is false for every world. This means that $\alpha \triangleright \beta$ is vacuously true when $\alpha$ is impossible or $\beta$ is necessary. Instead, when $\alpha$ is true in some world and $\beta$ is false is some world, (a) and (b) entail that there is a 10-free sphere where $\alpha$ is true in some world and $\beta$ is false in some world.

Validity is defined in terms of truth in a world in a model, and logical consequence is defined accordingly for every finite set of formulas $\alpha_{1}, \ldots, \alpha_{n}$ and every formula $\beta$.

Definition $4 \vDash \alpha$ iff $\alpha$ is true in every world in every model.

Definition $5 \alpha_{1}, \ldots \alpha_{n} \vDash \beta$ iff $\vDash\left(\alpha_{1} \wedge \ldots \wedge \alpha_{n}\right) \supset \beta$.

In the following sections we will employ these definitions to elucidate the logical features of the evidential interpretation. From now on, we will reason on an arbitrary model $\langle W, O, V\rangle$, and we will refer to conditions (a) and (b) of clause 6 of Definition 3.

\section{Some Relatively Uncontroversial Principles}

Let us start with five basic and relatively uncontentious principles that hold for $\supset$. The first is Modus Ponens: from a conditional and its antecedent one can infer its consequent. This is the simplest and most fundamental rule of inference involving conditionals, and most theorists of conditionals agree on its centrality. The evidential account validates Modus Ponens:

Fact $1 \alpha \triangleright \beta, \alpha \vDash \beta$

(Modus Ponens $\checkmark)$

Proof Assume that $[\alpha \triangleright \beta]_{w}=1$ and $[\alpha]_{w}=1$. Then, given (a), it follows that $[\beta]_{w}=1$, for $w$ is the closest world in which $\alpha$ is true.

The second principle, Superclassicality, says that a conditional is true if its consequent logically follows from its antecedent. This principle is very reasonable from 
the evidential point of view, for if $\beta$ logically follows from $\alpha$, then $\alpha$ provides a conclusive reason for accepting $\beta$.

Fact 2 If $\alpha \vDash \beta$, then $\vDash \alpha \triangleright \beta$

(Superclassicality $\checkmark)$

Proof Assume that $\alpha \vDash \beta$. Then, for every $w$, there is no $w^{\prime}$ such that $[\alpha]_{w^{\prime}}=1$ and $[\beta]_{w^{\prime}}=0$, which entails that (a) and (b) are both satisfied. Therefore, $[\alpha \triangleright \beta]_{w}=1$.

The third principle, Identity, says that every conditional whose consequent is identical to the antecedent is true. The evidential account validates this principle, as is plausible to expect:

Fact $3 \vDash \alpha \triangleright \alpha$

$($ Identity $\checkmark)$

Proof This directly follows from Fact 2, given that $\alpha \vDash \alpha$.

Two further principles, which involve the operators $\square$ and $\diamond$, are Necessary Consequent and Impossible Antecedent: a conditional is true if its consequent is necessary or its antecedent is impossible. The evidential account validates these two principles, given its treatment of vacuous truth:

Fact $4 \square \alpha \vDash \beta \triangleright \alpha$

$($ Necessary Consequent $\checkmark)$

Proof Assume that $[\square \alpha]_{w}=1$. Then by definition $[\beta \triangleright \alpha]_{w}=1$.

Fact $5 \sim \diamond \alpha \vDash \alpha \triangleright \beta$

(Impossible Antecedent $\checkmark)$

Proof Assume that $[\sim \diamond \alpha]_{w}=1$. Then by definition $[\alpha \triangleright \beta]_{w}=1$.

The five principles considered are relatively uncontentious in that most theories of conditionals preserve them. This does not mean, of course, that they are universally accepted. In particular, Necessary Consequent and Impossible Antecedent might be regarded as problematic, as it might be contended that there is a sense in which the antecedent of a vacuosuly true conditional is not relevant to its consequent. ${ }^{14}$

\footnotetext{
14 Priest (2008), pp. 72-77 spells out this objection. Relevant logicians are unwilling to grant Necessary Consequent and Impossible Antecedent, see Mares (2012). Another example is Gomes (2020), pp. 3-4, where these principles are rejected on the basis of considerations about the pragmatics of conditionals.
} 


\section{Some Highly Controversial Principles}

Now we will show that the evidential account invalidates some highly contentious principles that characterize $\supset$. In the material interpretation, False Antecedent and True Consequent hold, that is, the mere falsity of the antecedent or the mere truth of the consequent suffices for the truth of the conditional. This is commonly regarded as a reason to doubt the material interpretation. For example, it is quite implausible that the following sentences are true:

(12) If the Colisseum is not in Rome, then I won the lottery.

(13) If the Colisseum is in Rome, then I did not win the lottery. ${ }^{15}$

$\triangleright$ differs from $\supset$ in this respect. On the evidential account, (12) and (13) are false: it is easy to see that (12) fails the Ramsey Test and (13) fails the Reverse Ramsey Test. More generally, False Antecedent and True Consequent are invalid.

Fact $6 \sim \alpha \not \models \alpha \triangleright \beta$

(False Antecedent $\times$ )

Proof Suppose that $[\alpha]_{w}=0$ and that, for some $w^{\prime},[\alpha]_{w^{\prime}}=1,[\beta]_{w^{\prime}}=0$, and $w^{\prime} \in S$ for every $S \neq\{w\}$. In this case $[\sim \alpha]_{w}=1$. But $[\alpha \triangleright \beta]_{w}=0$, for $w^{\prime}$ violates (a).

Fact $7 \beta \not \models \alpha \triangleright \beta$

(True Consequent $\times$ )

Proof Suppose that $[\beta]_{w}=1$ and that, for some $w^{\prime},[\alpha]_{w^{\prime}}=1,[\beta]_{w^{\prime}}=0$, and $w^{\prime} \in S$ for every $S \neq\{w\}$. In this case $[\alpha \triangleright \beta]_{w}=0$, for $w^{\prime}$ violates (b).

A closely related principle that holds for $\supset$ is Linearity: for any $\alpha$ and $\beta$, either the conditional with antecedent $\alpha$ and consequent $\beta$ holds, or the conditional with antecedent $\beta$ and consequent $\alpha$ holds. For example, the following disjunction is true in the material interpretation:

(14) Either if it is snowing then I will win the lottery or if I will win the lottery then it is snowing

Again, $\triangleright$ differs from $\supset$ in this respect. On the evidential account, (14) is false because in each disjunct the antecedent and the consequent are not related in the right way. More generally, the evidential account invalidates Linearity.

Fact $8 \not \models(\alpha \triangleright \beta) \vee(\beta \triangleright \alpha)$

(Linearity $\times)$

${ }^{15}$ Edgington (2001), Section 2.3, presents False Antecedent and True Consequent as "the best-known objection to the material account". 
Proof Suppose that $[\alpha]_{w}=0$ and $[\beta]_{w}=0$. Let $w^{\prime}$ and $w^{\prime \prime}$ be such that $[\alpha]_{w^{\prime}}=1$, $[\beta]_{w^{\prime}}=0,[\alpha]_{w^{\prime \prime}}=0,[\beta]_{w^{\prime \prime}}=1$, and that $w^{\prime} \in S$ and $w^{\prime \prime} \in S$ for every $S \neq\{w\}$. In this case $[\alpha \triangleright \beta]_{w}=0$ because $w^{\prime}$ violates (a). Moreover, $[\beta \triangleright \alpha]_{w}=0$ because $w^{\prime \prime}$ violates (a).

Another closely related principle that holds for $\supset$ is Conditional Proof: if $\alpha$, together with a set of premises $\Gamma$, entails $\beta$, then the conditional with antecedent $\alpha$ and consequent $\beta$ follows from $\Gamma$. This principle entails False Antecedent and True Consequent, so it is inconsistent with any account that rejects False Antecedent and True Consequent.

Fact 9 Not: if $\Gamma, \alpha \vDash \beta$, then $\Gamma \vDash \alpha \triangleright \beta$

(Conditional Proof $\times$ )

Proof Suppose that the following holds: if $\Gamma, \alpha \vDash \beta$, then $\Gamma \vDash \alpha \triangleright \beta$. Since $\sim \alpha, \alpha \vDash \beta$ and $\beta, \alpha \vDash \beta$, we get that $\sim \alpha \vDash \alpha \triangleright \beta$ and $\beta \vDash \alpha \triangleright \beta$, contrary to Facts 6 and 7 .

Two further properties of $\supset$ are widely regarded as undesirable. One is Monotonicity, the principle according to which one can always strengthen the antecedent of a conditional by adding any conjunct. The other is Transitivity, the principle according to which a conditional with antecedent $\alpha$ and consequent $\beta$ and a conditional with antecedent $\beta$ and consequent $\gamma$ together entail the conditional with antecedent $\alpha$ and consequent $\gamma$. Examples such as the following, due to Adams, have been taken to show - and plausibly so, we think - that conditionals as they are used in ordinary language are neither monotonic nor transitive:

If Brown wins the election, Smith will retire to private life. Therefore, if Smith dies before the election and Brown wins it, Smith will retire to private life.

If Brown wins the election, Smith will retire to private life. If Smith dies before the election,

Brown will win it. Therefore, if Smith dies before the election, then he will retire to private life. ${ }^{16}$

The evidential account can explain the apparent invalidity of these arguments. It is possible that the premise of the first argument is true but its conclusion is false, for only the former passes the Ramsey Test. Similarly, it is possible that the premises of the second argument are true but its conclusion is false, for only the former pass the Ramsey Test. More generally, the evidential account invalidates Monotonicity and Transitivity.

Fact $10 \alpha \triangleright \gamma \not \models(\alpha \wedge \beta) \triangleright \gamma$

(Monotonicity $\times)$

Proof Suppose that $[\alpha \triangleright \gamma]_{w}=1$ and, for some $S$, there is no $w^{\prime} \in S$ such that $[\beta]_{w^{\prime}}=1$. Suppose also that outside $S$ there is a $w^{\prime \prime}$ such that $[\alpha]_{w^{\prime \prime}}=1,[\beta]_{w^{\prime \prime}}=1$, $[\gamma]_{w^{\prime \prime}}=0$, and $w^{\prime \prime}$ belongs to every $S^{\prime}$ bigger than $S$. In this case $[(\alpha \wedge \beta) \triangleright \gamma]_{w}=0$ because $w^{\prime \prime}$ violates (a).

16 Adams (1965), p. 166. 
Proof If Transitivity were valid, then Monotonicity would be valid as well, contrary to Fact 10 . Since $[(\alpha \wedge \gamma) \triangleright \alpha]_{w}=1$ by Fact 2 , if one assumes that $[\alpha \triangleright \beta]_{w}=1$, by Transitivity one gets that $[(\alpha \wedge \gamma) \triangleright \beta]_{w}=1$.

\section{Contraposition and Right Weakening}

The facts outlined in Sects. 4 and 5 are results on which most non-material accounts of conditionals tend to converge: Modus Ponens, Superclassicality, Identity, Necessary Consequent, and Impossible Antecedent are widely accepted as sound, while False Antecedent, True Consequent, Linearity, Monotonicity, and Transitivity are widely regarded as counterintuitive. The principles considered in this section and in the next two, instead, are more divisive. As we shall see, the evidential account crucially differs from other non-material accounts with respect to these principles.

The evidential account preserves Contraposition, the principle according to which a conditional with antecedent $\alpha$ and consequent $\beta$ entails the conditional with antecedent $\sim \beta$ and consequent $\sim \alpha$. To illustrate, consider the inference from (1) to (15):

(15) If it shrinks, then it is not pure cashmere

This inference seems valid, and the same goes for similar inferences that involve (2) and (3) as premises. More generally, Contraposition holds for $\triangleright$ :

Fact $12 \alpha \triangleright \beta \vDash \sim \beta \triangleright \sim \alpha$

$($ Contraposition $\checkmark)$

Proof Assume that $[\alpha \triangleright \beta]_{w}=1$. Then (a), for every $w^{\prime}$, if $[\alpha]_{w^{\prime}}=1$ and there are no $w^{\prime \prime}$ and $S$ such that $w^{\prime \prime} \in S, w^{\prime} \notin S$, and $[\alpha]_{w^{\prime \prime}}=1$, then $[\beta]_{w^{\prime}}=1$, and (b) for every $w^{\prime}$, if $[\beta]_{w^{\prime}}=0$ and there are no $w^{\prime \prime}$ and $S$ such that $w^{\prime \prime} \in S, w^{\prime} \notin S$, and $[\beta]_{w^{\prime \prime}}=0$, then $[\alpha]_{w^{\prime}}=0$. (a) and (b) are respectively (b) and (a) for $\sim \beta \triangleright \sim \alpha$. Therefore, $[\sim \beta \triangleright \sim \alpha]_{w}=1$.

This is a distinctive feature of the evidential account. Unlike the principles considered in the previous two sections, Contraposition is neither widely accepted nor widely rejected. Some theorists of conditionals regard it as counterintuitive. Here is a classical example due to Stalnaker:

'If the US halts the bombing, then North Vietnam will not agree to negotiate'. A person would believe that this statement is true if he thought that the North Vietnamese were determined to 
press for a complete withdrawal of US troops. But he would surely deny the contrapositive, 'If North Vietnam agrees to negotiate, then the US will not have halted the bombing'. ${ }^{17}$

However, these examples can hardly prove that Contraposition fails in the evidential interpretation. As has been noted by Lycan, Bennett, Gomes, and others, the alleged counterexamples to Contraposition typically involve a concessive reading of the premise. So, they loose their grip on any interpretation which rules out such a reading. ${ }^{18}$ This is precisely the case in point: concessive conditionals are false from the evidential point of view. The conditional 'If the US halts the bombing, then North Vietnam will not agree to negotiate' does not pass the Reverse Ramsey Test, for it is not the case that the closest worlds in which North Vietnam will agree to negotiate are worlds in which the US keep bombing. If North Vietnam will not agree to negotiate, it is not because the US halts the bombing, but in spite of that fact. More generally, insofar as the alleged counterexamples to Contraposition involve a concessive reading of the premise, they do not work in the evidential interpretation because their premise turns out to be false.

A closely related fact concerns Right Weakening, the principle according to which, if $\gamma$ logically follows from $\beta$, then a conditional with antecedent $\alpha$ and consequent $\beta$ entails the conditional with antecedent $\alpha$ and consequent $\gamma$. This principle does not hold for $\triangleright$. Consider the following example, taken from Rott:

It makes perfect sense to say 'If you pay an extra fee, your letter will be delivered by express', because the fee will buy you a special service. But it sounds odd to say 'If you pay an extra fee, your letter will be delivered', because the letter would be delivered anyway, even if you did not pay the extra fee. ${ }^{19}$

The first conditional is definitely plausible from the evidential point of view. There is a clear sense in which the payment of the extra fee constitutes a reason for thinking that the letter will be delivered by express. However, the second conditional is not equally plausible. The payment of the extra fee, at least in some sense, does not constitute a reason for thinking that the letter will be delivered: if you expect that the letter will be delivered, it is not in virtue of the extra fee. The key to our understanding of this difference is the Reverse Ramsey Test. Among the worlds in which the letter will not be delivered by express, those in which you did not pay the extra fee are closer than those in which you paid it. But there is no reason to think that, among the worlds in which the letter will not be delivered, those in which you did not pay the extra fee are closer than those in which you paid it. The closest worlds in which the letter is not delivered will rather have other kinds of features, like the occurrence of some accident, in virtue of which the delivery failed altogether, regardless of your payment of the extra fee. Therefore, it is consistent with the evidential account to say that the first conditional is true but the second is false. And since the consequent of the first-once naturally

\footnotetext{
17 Stalnaker (1991), p. 39.

18 Lycan (2001), p. 34, Bennett (2003), pp. 32 and 143-144, Gomes (2019).

19 Rott (2020), p. 6.
} 
formalized in a propositional language-entails the consequent of the second, this means that the evidential account invalidates Right Weakening.

Fact 13 Not: if $\beta \vDash \gamma$, then $\alpha \triangleright \beta \vDash \alpha \triangleright \gamma$

(Right Weakening $\times)$

Proof Right Weakening fails because it entails Monotonicity, given Contraposition. Assume that $[\alpha \triangleright \gamma]_{w}=1$. By Fact 12, this entails that $[\sim \gamma \triangleright \sim \alpha]_{w}=1$. Since $\sim \alpha \vDash \sim \alpha \vee \sim \beta$, if Right Weakening were valid, we would get that $[\sim \gamma \triangleright(\sim \alpha \vee \sim \beta)]_{w}=1$, and so that $[\sim(\sim \alpha \vee \sim \beta) \triangleright \sim \sim \gamma]_{w}=1$ again by Fact 12 . Since $\sim(\sim \alpha \vee \sim \beta) \triangleright \sim \sim \gamma$ is logically equivalent to $(\alpha \wedge \beta) \triangleright \gamma$, this means that $[(\alpha \wedge \beta) \triangleright \gamma]_{w}=1$, contrary to Fact 10 .

This proof makes explicit the connection between Contraposition and Right Weakening: if Monotonicity fails, then either Contraposition or Right Weakening must fail as well. This is why Facts 12 and 13 are closely related.

\section{Conditional Excluded Middle and Conjunctive Sufficiency}

One rather debated principle is Conditional Excluded Middle: for every $\alpha$ and $\beta$, either the conditional with antecedent $\alpha$ and consequent $\beta$ holds, or the conditional with antecedent $\alpha$ and consequent $\sim \beta$ holds. Some non-material accounts of conditionals retain this principle, while others deny it. The evidential account belongs to the second category. Consider the following sentences:

(16) If planet nine exists, then the EU will collapse within 5 years

(17) If planet nine exists, then the EU will not collapse within 5 years

Since there is no relation at all between the existence of planet nine and the collapse of the EU, it is quite reasonable to say that (16) and (17) are both false. So, the same goes for the disjunction of (16) and (17). This is exactly what the evidential account predicts, given that neither (16) nor (17) pass the Chrysippus Test. More generally, Conditional Excluded Middle does not hold for $\triangleright$ :

Fact $14 \not \models(\alpha \triangleright \beta) \vee(\alpha \triangleright \sim \beta)$

(Conditional Excluded Middle $\times$ )

Proof Suppose that $[\alpha]_{w}=1$ and $[\beta]_{w}=0$. Let $w^{\prime}$ be such that $[\alpha]_{w^{\prime}}=1,[\beta]_{w^{\prime}}=1$, and $w^{\prime} \in S$ for every $S \neq\{w\}$. In this case $[\alpha \triangleright \beta]_{w}=0$ because $w$ violates both (a) and (b). Moreover, $[\alpha \triangleright \sim \beta]_{w}=0$, for $w^{\prime}$ violates (b).

Another debated principle that fails on the evidential account is Conjunctive Sufficiency, according to which any conjunction entails the corresponding conditional. Even supposing that the antecedent and the consequent of (16) are both true, it does not follow that (16) is true. The same goes for (17). We take the 
failure of Conjunctive Sufficiency to be a desirable result. If $\alpha$ and $\beta$ are totally unrelated, it is definitely false that $\alpha$ provides a reason to accept $\beta$, or that $\beta$ can be inferred from $\alpha$.

Fact $15 \alpha \wedge \beta \not \models \alpha \triangleright \beta$

(Conjunctive Sufficiency $\times$ )

Proof Suppose that $[\alpha]_{w}=1,[\beta]_{w}=1$, and for some $w^{\prime},[\alpha]_{w^{\prime}}=1,[\beta]_{w^{\prime}}=0$, and $w^{\prime} \in S$ for every $S \neq\{w\}$. In this case $[\alpha \wedge \beta]_{w}=1$. But $[\alpha \triangleright \beta]_{w}=0$, for $w^{\prime}$ violates (b).

Conditional Excluded Middle and Conjunctive Sufficiency somehow stem from the very same assumption, namely, that the negation of a conditional with antecedent $\alpha$ and consequent $\beta$ entails the conditional with antecedent $\alpha$ and consequent $\sim \beta$. If this assumption is granted, then Conditional Excluded Middle straightforwardly follows from Excluded Middle. Moreover, Conjunctive Sufficiency can be derived by reductio, for if one assumes $\alpha \wedge \beta$ and the negation of the conditional with antecedent $\alpha$ and consequent $\beta$, one gets $\beta$ and $\sim \beta$. The evidential account rejects this key assumption: $\sim(\alpha \triangleright \beta)$ does not entail $\alpha \triangleright \sim \beta$. Therefore, $(\alpha \triangleright \beta) \vee(\alpha \triangleright \sim \beta)$ does not follow from $(\alpha \triangleright \beta) \vee \sim(\alpha \triangleright \beta)$, and $\alpha \triangleright \beta$ is not derivable from $\alpha \wedge \beta$ by reductio.

Fact 15 is particularly interesting because it shows that a principled distinction can be drawn between two claims that are usually conflated. One is centering, understood as a condition on the system of spheres that is based on a metaphysical assumption. The other is Conjunctive Sufficiency, the logical rule just discussed. In the semantic framework offered by Lewis, if one assumes centering, one gets Conjunctive Sufficiency. As Lewis himself suggests, one can avoid this result by replacing centering with weak centering, that is, by replacing clause 2 of Definition 1 with the condition that $w$ belongs to every sphere around $w$, without requiring that the innermost sphere is a singleton. ${ }^{20}$ This is why in the literature on conditionals it is quite common to talk about Conjunctive Sufficiency and centering as if they were the same thing. However, this coincidence breaks down in our semantic framework: even if one assumes centering, as in Definition 1, one does not get Conjunctive Sufficiency. This shows that the question whether Conjunctive Sufficiency holds does not reduce to the choice between centering and weak centering. As a matter of fact centering plays no essential role in our account, for the Chrysippus Test could be phrased equally well by assuming weak centering. But independently of the considerations that may lead one to choose between centering and weak centering, the point is that the choice has no effect on Conjunctive Sufficiency.

${ }^{20}$ Lewis (1973), p. 29 


\section{Connexivity}

The last set of principles that we will consider has been extensively discussed in relation to connexive logics. Connexive logics are characterized by two main theses, Aristotle's Thesis and Boethius' Thesis. The former says that the negation of a conditional with antecedent $\alpha$ and consequent $\sim \alpha$ is always true. The latter says that if a conditional with antecedent $\alpha$ and consequent $\beta$ is true, then the conditional with antecedent $\alpha$ and consequent $\sim \beta$ is false. Some connexivists have suggested that an account of conditionals based on a suitable reading of Chrysippus's claim can capture the intuitive esssence of Aristotle's Thesis and Boethius' Thesis, and we are inclined to agree with them. ${ }^{21}$

We believe that the idea of connexivity rests on a solid intuition, although we doubt that Aristotle's Thesis and Boethius' Thesis hold unrestrictedly. Consider the following sentence:

(18) If it is snowing, then it is not snowing

It is natural to feel that there is something wrong in (18), and this feeling can hardly be dispelled by noting that (18) is false if it is snowing, as the material interpretation predicts. So we find it plausible to say that the negation of (18) is true no matter whether it is snowing or not. This does not mean, however, that every conditional whose consequent is the negation of the antecedent is intuitively false. For example, we have no clear intuitions about (19):

(19) If it is not the case that either it is snowing or it is not snowing, then either it is snowing or it is not snowing

Similar considerations hold for Boethius' thesis. We find it plausible to say that if (3) is true, the following conditional is false:

(20) If it is snowing, then it is not cold

However, it is not obvious that the same holds for any two conditionals of the same form. As far as we can see, it is reasonable to think that (21) and (22) are both true:

(21) If it is snowing and it is not snowing, then it is snowing

(22) If it is snowing and it is not snowing, then it is not snowing

More generally, we think that Aristotle's thesis and Boethius' thesis are plausible insofar as they entail two weaker claims. One is Restricted Aristotle's Thesis: whenever $\alpha$ is possible, the negation of the conditional with antecedent $\alpha$ and consequent $\sim \alpha$ is true. The other is Restricted Abelard's Thesis: whenever $\alpha$ is possible, the

${ }^{21}$ McCall (2012) and Wansing (2020) suggest that the idea of connexivity goes back to Chrysippus. 
negation of the conjunction of the conditional with antecedent $\alpha$ and consequent $\beta$ and the conditional with antecedent $\alpha$ and consequent $\sim \beta$ is true. This is a restricted version of Abelard's First Principle - the negation of the conjunction of a conditional with antecedent $\alpha$ and consequent $\beta$ and the conditional with antecedent $\alpha$ and consequent $\sim \beta$ is always true-which is in turn a conjunctive version of Boethius' Thesis. $^{22}$

A third connexive thesis that deserves attention is Aristotle's Second Thesis: the negation of the conjunction of a conditional with antecedent $\alpha$ and consequent $\beta$ and the conditional with antecedent $\sim \alpha$ and consequent $\beta$ is always true. ${ }^{23}$ As in the case of Aristotle's Thesis and Boethius' Thesis, we think that this thesis is plausible as long as we restrict consideration to non-vacuously true conditionals. For example, it reasonable to deny the conjunction of (1) and (23):

(23) If it is not pure cashmere, it will not shrink

Yet it is not equally reasonable to deny the conjunction of (24) and (25):

(24) If it is snowing, then either it is snowing or it is not snowing

(25) If it is not snowing, then either it is snowing or it is not snowing

More generally, we think that Aristotle's Second Thesis is plausible insofar as it entails a weaker principle, Restricted Aristotle's Second Thesis: whenever $\beta$ is not necessary, the negation of the conjunction of a conditional with antecedent $\alpha$ and consequent $\beta$ and the conditional with antecedent $\sim \alpha$ and consequent $\beta$ is true.

The evidential account yields exactly what we would expect. First, (18) can be denied because it blatantly fails both the Ramsey Test and the Reverse Ramsey Test. Second, the conjunction of (3) and (20) can be denied because if (3) is true, then (20) is false: it is impossible that both (3) and (20) pass the Ramsey Test. Third, the conjunction of (1) and (23) can be denied because if (1) is true, then (23) is false: it is impossible that both (1) and (23) pass the Reverse Ramsey Test. Instead, (19), (21), and (22) are vacuously true because their antecedent is impossible, and similarly (24) and (25) are vacuously true because their consequent is necessary. ${ }^{24}$

Now we will prove that Restricted Aristotle's Thesis, Restricted Abelard's Thesis, and Restricted Aristotle's Second Thesis hold for $\triangleright$. In order to do so, we will prove a stronger principle, Restricted Selectivity ${ }^{25}$ :

Fact 16 If $\beta \vDash \sim \gamma$, then $\diamond \alpha, \alpha \triangleright \beta \vDash \sim(\alpha \triangleright \gamma)$

(Restricted Selectivity $\checkmark)$

\footnotetext{
22 More precisely, it is a conjunctive version of Weak Boethius' Thesis, see Wansing (2020). Abelard's First Principle is what Angell (1962) called "principle of subjunctive contrariety". Unterhuber (2016), Lenzen (2019b), Kapsner (2019), Iacona (2020), consider restricted versions of connexive principles.

23 See McCall (2012), Estrada-Gonzáles and Ramírez-Cámara (2016), pp. 346-348.

${ }^{24}$ In this respect, a possible divergence from Chrysippus' original view must be acknowledged, for it is a controversial matter whether he regarded conditionals with impossible antecedents as true.

25 See Huber (2007), p. 531.
} 
Proof Assume that $\beta \vDash \sim \gamma,[\diamond \alpha]_{w}=1$, and $[\alpha \triangleright \beta]_{w}=1$. Since $[\nabla \alpha]_{w}=1, \alpha$ is true in some worlds. Since $[\alpha \triangleright \beta]_{w}=1$, for every $w^{\prime}$ such that $[\alpha]_{w^{\prime}}=1$ and there are no $w^{\prime \prime}$ and $S$ such that $w^{\prime \prime} \in S, w^{\prime} \notin S$, and $[\alpha]_{w^{\prime \prime}}=1$, then $[\beta]_{w^{\prime}}=1$. Since $\beta \vDash \sim \gamma$, it follows that $[\sim \gamma]_{w^{\prime}}=1$. So $[\gamma]_{w^{\prime}}=0$. Therefore, $[\alpha \triangleright \gamma]_{w}=0$, and consequently $[\sim(\alpha \triangleright \gamma)]_{w}=1$.

Fact $17 \diamond \alpha \vDash \sim((\alpha \triangleright \beta) \wedge(\alpha \triangleright \sim \beta))$

(Restricted Abelard's Thesis $\checkmark$ )

Proof Assume that $[\diamond \alpha]_{w}=1$. Since $\beta \vDash \sim \sim \beta$, if $[\alpha \triangleright \beta]_{w}=1$, by Fact 16 we get that $[\sim(\alpha \triangleright \sim \beta)]_{w}=1$, hence that $[\alpha \triangleright \sim \beta]_{w}=0$. Therefore, $[(\alpha \triangleright \beta) \wedge(\alpha \triangleright \sim \beta)]_{w}=0$.

Fact $18 \diamond \alpha \vDash \sim(\alpha \triangleright \sim \alpha)$

(Restricted Aristotle's Thesis $\checkmark$ )

Proof Assume that $[\nabla \alpha]_{w}=1$. Since $[\alpha \triangleright \alpha]_{w}=1$ by Fact 3 , we get that $[\alpha \triangleright \sim \alpha]_{w}=0$, because Fact 17 entails that $[(\alpha \triangleright \alpha) \wedge(\alpha \triangleright \sim \alpha)]_{w}=0$. Therefore, $[\sim(\alpha \triangleright \sim \alpha)]_{w}=1$.

Fact $19 \diamond \sim \beta \vDash \sim((\alpha \triangleright \beta) \wedge(\sim \alpha \triangleright \beta)) \quad$ (Restricted Aristotle's Second Thesis $\checkmark)$

Proof Assume that $[\diamond \sim \beta]_{w}=1$. If $[\alpha \triangleright \beta]_{w}=1$, then $[\sim \beta \triangleright \sim \alpha]_{w}=1$ by Fact 12. So, $[\sim(\sim \beta \triangleright \alpha)]_{w}=1$ by Fact 16 , given that $\sim \alpha \vDash \sim \alpha$. This means that $[\sim \beta \triangleright \alpha]_{w}=0$, and consequently that $[\sim \alpha \triangleright \beta]_{w}=0$, for $\sim \beta \triangleright \alpha$ and $\sim \alpha \triangleright \beta$ have the same truth conditions (switch (a) and (b) as in the proof of Fact 12). Therefore, $[(\alpha \triangleright \beta) \wedge(\sim \alpha \triangleright \beta)]_{w}=0$.

It is important to note that the four principles just considered, unlike their unrestricted counterparts, imply no revision of classical logic. This turns out clear if one thinks that the same principles hold in the strict interpretation simply as theorems of standard modal logic. While the systems of connexive logic are typically contraclassical, in that they are neither subsystems nor extensions of classical logic, the form of restricted connexivity suggested here can be preserved without adopting such a system. ${ }^{26}$

\section{Comparisons}

The facts set out in Sects. 4-8 delineate the distinctive logical profile of the evidential interpretation. The evidential interpretation substantially departs from the material interpretation in that it invalidates False Antecedent, True Consequent, Linearity, Conditional Proof, Monotonicity, Transitivity, and Right Weakening, while it validates Restricted Selectivity, Restricted Aristotle's Thesis, Restricted Abelard's

${ }^{26}$ Iacona (2020) discusses restricted connexivity in the framework of the strict interpretation. 
Thesis, and Restricted Aristotle's Second thesis. Moreover, it differs from the strict interpretation because the latter retains Monotonicity, Transitivity, and Right Weakening. ${ }^{27}$ In terms of strength, the evidential interpretation lies between these two interpretations. If $\alpha \_\beta$ abbreviates $\square(\alpha \supset \beta)$, this is to say that $\alpha \_\beta$ entails $\alpha \triangleright \beta$ and $\alpha \triangleright \beta$ entails $\alpha \supset \beta$.

Fact $20 \alpha-\beta \beta \vDash \triangleright \beta$

(Strict to Evidential $\checkmark$ )

Proof Assume that $\left[\alpha_{-3} \beta\right]_{w}=1$. Then, for every $w^{\prime}$, if $[\alpha]_{w^{\prime}}=1$, then $[\beta]_{w^{\prime}}=1$, and for every $w^{\prime}$, if $[\beta]_{w^{\prime}}=0$, then $[\alpha]_{w^{\prime}}=0$. Therefore, $[\alpha \triangleright \beta]_{w}=1$.

Fact $21 \alpha \triangleright \beta \vDash \alpha \supset \beta$

(Evidential to Material $\checkmark$ )

Proof This follows directly from Fact 1.

More importantly, the evidential interpretation differs from the accounts of conditionals advocated by Adams, Stalnaker, Lewis, and others. We will use the umbrella term 'suppositional interpretation' for these accounts, regardless of the specific traits that characterize each of them, and we will use the symbol $\Rightarrow$ accordingly. Broadly speaking, $\alpha \Rightarrow \beta$ means that $\beta$ is credible enough given $\alpha$. That is, on the supposition that $\alpha$ holds, there are good chances that $\beta$ holds. Just as the evidential interpretation, the suppositional interpretation lies between the strict interpretation and the material interpretation. But it is weaker than the evidential interpretation: $\alpha \triangleright \beta$ entails $\alpha \Rightarrow \beta$, but not the other way round. Truth in the suppositional sense is defined solely in terms of the Ramsey Test, so it holds no matter whether the Reverse Ramsey Test is satisfied.

The relation between the evidential interpretation and the suppositional interpretation can be stated more precisely by assuming that $[\alpha \Rightarrow \beta]_{w}=1$ if and only if (a) is satisfied. On this assumption, we have the following equivalence:

Fact $22[\alpha \triangleright \beta]_{w}=1$ iff $[(\alpha \Rightarrow \beta) \wedge(\sim \beta \Rightarrow \sim \alpha)]_{w}=1$

Proof Assume that $[\alpha \triangleright \beta]_{w}=1$. Since (a) holds for $\alpha$ and $\beta,[\alpha \Rightarrow \beta]_{w}=1$. Since (b) holds for $\alpha$ and $\beta$, (a) holds for $\sim \beta$ and $\sim \alpha$, hence $[\sim \beta \Rightarrow \sim \alpha]_{w}=1$. Therefore, $[(\alpha \Rightarrow \beta) \wedge(\sim \beta \Rightarrow \sim \alpha)]_{w}=1$. The proof of the right-to-left direction is similar.

Fact 22 shows that $\triangleright$ is definable in terms of $\Rightarrow$. The opposite is also true, although less trivial, that is, $\Rightarrow$ is definable in terms of $\triangleright .^{28}$

\footnotetext{
${ }^{27}$ Influential analyses of non-material monotonic conditionals have been sometimes integrated in socalled dynamic semantics. A thorough comparison with these approaches would reveal even more divergences from ours. For instance, in Veltman's theory, presented in Veltman (1986), True Consequent is valid while Modus Tollens is not.

${ }^{28}$ We owe this equivalence result to Eric Raidl, who provides an extensive analysis of the mutual definability of different kinds of conditional in Raidl (2020).
} 
Fact $23[\alpha \Rightarrow \beta]_{w}=1$ iff $[(\alpha \wedge \beta) \vee(\alpha \triangleright(\alpha \wedge \beta))]_{w}=1$

Proof Assume that $[(\alpha \wedge \beta) \vee(\alpha \triangleright(\alpha \wedge \beta))]_{w}=1$. If $[\alpha \wedge \beta]_{w}=1$, then $w$ verifies both the antecedent and the consequent of (a), while every other world falsifies its antecedent. Therefore, $[\alpha \Rightarrow \beta]_{w}=1$. If $[\alpha \triangleright(\alpha \wedge \beta)]_{w}=1$, then $[\alpha \Rightarrow(\alpha \wedge \beta)]_{w}=1$, which means that $\alpha \wedge \beta$ is true in the closest worlds in which $\alpha$ is true. It follows that $\beta$ is true in the closest worlds in which $\alpha$ is true, that is, $[\alpha \Rightarrow \beta]_{w}=1$.

Now assume that $[\alpha \Rightarrow \beta]_{w}=1$. Then either $[\alpha]_{w}=1$ or $[\alpha]_{w}=0$. If $[\alpha]_{w}=1$, then $[\beta]_{w}=1$, for $w$ verifies the antecedent of (a). It follows that $[\alpha \wedge \beta]_{w}=1$, and consequently that $[(\alpha \wedge \beta) \vee(\alpha \triangleright(\alpha \wedge \beta))]_{w}=1$. If $[\alpha]_{w}=0$, then $[\sim \alpha]_{w}=1$, so $[\sim \alpha \vee \sim \beta]_{w}=1$. This entails that $[(\sim \alpha \vee \sim \beta) \Rightarrow \sim \alpha]_{w}=1$, for $w$ verifies both the antecedent and the consequent of (a), while every other world falsifies its antecedent. It follows that $[\sim(\alpha \wedge \beta) \Rightarrow \sim \alpha]_{w}=1$, given that $\sim \alpha \vee \sim \beta$ is logically equivalent to $\sim(\alpha \wedge \beta)$. Moreover, the assumption that $[\alpha \Rightarrow \beta]_{w}=1$ entails that $[\alpha \Rightarrow(\alpha \wedge \beta)]_{w}=1$ : if $\beta$ is true in the closest worlds in which $\alpha$ is true, so is $\alpha \wedge \beta$. By fact 22 we get that $[\alpha \triangleright(\alpha \wedge \beta)]_{w}=1$, hence that $[(\alpha \wedge \beta) \vee(\alpha \triangleright(\alpha \wedge \beta))]_{w}=1$.

The contrast between $\triangleright$ and $\Rightarrow$ emerges clearly if we consider the principles discussed in Sects. 6-8. As explained in Sect. 6, Contraposition holds for $\triangleright$. Instead, it does not hold for $\Rightarrow$. The examples that are usually taken to show that Contraposition fails, such as the inference about the US and North Vietnam, include concessive conditionals as premises, so they do not work if conditionals are understood evidentially. But the same examples work if conditionals are understood suppositionally, for their premises turn out to be true.

Right Weakening produces opposite results: it holds for $\Rightarrow$ but not for $\triangleright$. The examples that can rightfully be taken to show that Right Weakening fails, such as the inference about the letter, work only if conditionals are understood evidentially, for they involve conclusions that are false in the evidential sense. Instead, the same examples do not work if conditionals are understood suppositionally, for their conclusions turn out to be true.

As explained in Sect. 7, Conditional Excluded Middle and Conjunctive Sufficiency do not hold for $\triangleright$. Instead, both principles hold for $\Rightarrow$. Although the core idea of the suppositional interpretation-Ramsey's original idea-by itself does not entail Conditional Excluded Middle, and can be developed in the way suggested by Lewis, a natural reading of that idea accords perfectly well with Conditional Excluded Middle: to say that $\beta$ does not hold on the supposition that $\alpha$ holds is to say that $\sim \beta$ holds on that supposition, so denying $\alpha \Rightarrow \beta$ amounts to asserting $\alpha \Rightarrow \sim \beta$. This is the reading adopted by Adams and Stalnaker. Conjunctive Sufficiency is valid as well: if $\alpha$ and $\beta$ actually hold, then obviously there are good chances that $\beta$ holds on the supposition that $\alpha$ holds.

Finally, $\triangleright$ and $\Rightarrow$ agree on Restricted Aristotle's Thesis and Restricted Abelard's Thesis, but they differ with respect to Restricted Aristotle's Second Thesis, as the latter does not hold for $\Rightarrow$. Consider (11). Since (11) is acceptable in the suppositional sense, if we replace its antecedent with 'You don't drink a beer' we obtain a conditional which is also acceptable in the suppositional sense: there are good chances that its consequent holds on the supposition that its antecedent holds. 
Not only the evidential account differs from the suppositional theories of conditionals in the way explained, but it also differs in important respects from some recent attempts to provide a non-monotonic theory of conditionals based on the notion of support. One is Rott's interesting treatment of "difference-making" conditionals, which adopts a strengthened version of the Ramsey Test in the context of the classical theory of belief revision. Rott's account, like ours, invalidates Monotonicity and Right Weakening. Unlike ours, however, it does not retain Contraposition, even though Contraposition is consistent with the rejection of Monotonicity, provided that Right Weakening fails. As far as we can see, this result has no obvious rationale. Once the concessive reading of 'if then' is ruled out, and the alleged counterexamples such as that considered in Sect. 6 loose their grip, it is no longer clear what reason one may have for rejecting Contraposition. ${ }^{29}$

The other example is Douven's epistemic analysis of conditionals, which relies on a notion of evidential support defined in terms of degrees of belief. Douven's account yields a considerably weak logic, in which several widely accepted principles, including Modus Ponens, turn out to be invalid. Therefore, it significantly differs from our account, which preserves Modus Ponens and other basic principles. ${ }^{30}$

The following table summarizes what has been said so far about the logical profile of the evidential interpretation, as it displays some key similarities and differences between $\supset, \Rightarrow, \triangleright$, and $\_$.

\begin{tabular}{lcccc}
\hline & $\supset$ & $\Rightarrow$ & $\triangleright$ & -3 \\
\hline Modus Ponens & $\checkmark$ & $\checkmark$ & $\checkmark$ & $\checkmark$ \\
Superclassicality & $\checkmark$ & $\checkmark$ & $\checkmark$ & $\checkmark$ \\
Identity & $\checkmark$ & $\checkmark$ & $\checkmark$ & $\checkmark$ \\
Necessary Consequent & $\checkmark$ & $\checkmark$ & $\checkmark$ & $\checkmark$ \\
Impossible Antecedent & $\checkmark$ & $\checkmark$ & $\checkmark$ & $\checkmark$ \\
False Antecedent & $\checkmark$ & $\times$ & $\times$ & $\times$ \\
True Consequent & $\checkmark$ & $\times$ & $\times$ & $\times$ \\
Linearity & $\checkmark$ & $\times$ & $\times$ & $\times$ \\
Conditional Proof & $\checkmark$ & $\times$ & $\times$ & $\times$ \\
Monotonicity & $\checkmark$ & $\times$ & $\times$ & $\checkmark$ \\
Transitivity & $\checkmark$ & $\times$ & $\times$ & $\checkmark$ \\
Contraposition & $\checkmark$ & $\times$ & $\checkmark$ & $\checkmark$ \\
Right Weakening & $\checkmark$ & $\checkmark$ & $\times$ & $\checkmark$ \\
Conditional Excluded Middle & $\checkmark$ & $\checkmark$ & $\times$ & $\times$ \\
Conjunctive Sufficiency & $\checkmark$ & $\checkmark$ & $\times$ & $\times$ \\
Restricted Selectivity & $\times$ & $\checkmark$ & $\checkmark$ & $\checkmark$ \\
Restricted Abelard's Thesis & $\times$ & $\checkmark$ & $\checkmark$ & $\checkmark$ \\
Restricted Aristotle's Thesis & $\times$ & $\checkmark$ & $\checkmark$ & $\checkmark$ \\
Restricted Aristotle's Second Thesis & $\times$ & $\times$ & $\checkmark$ & $\checkmark$ \\
\hline
\end{tabular}

${ }^{29}$ Rott (2020). The account provided in Berto and Özgün (2020) also entails failure of Contraposition.

${ }^{30}$ Douven (2016), ch. 5. 
We have reason to conjecture that the evidential account can effectively be developed at the proof-theoretic level, namely, that there is an axiom system for $\triangleright$ that preserves the results listed in the third column above and is sound and complete with respect to the semantics outlined in Sect. 3. But we will leave the proof of this conjecture to another work.

\section{Truth and Assertibility}

In this paper we pursue a truth-conditional approach to conditionals, that is, we define the evidential interpretation by specifying the conditions under which a conditional is true on that interpretation. More specifically, we adopt a standard characterization of truth relative to worlds, and define logical consequence accordingly.

This is not the only possibility, however. As is well known, an alternative route is available, whereby truth conditions are deliberately avoided and the semantics is framed in terms of assertibility. According to Adams, who suggested this route, a conditional with antecedent $\alpha$ and consequent $\beta$ is assertible to the extent that the probability of $\beta$ conditional on $\alpha$ is high. In this analysis, the degree of assertibility of the conditional relative to a probability distribution $P$ is $P(\beta \mid \alpha)$, and its corresponding degree of uncertainty is 1 minus that degree. Apart from specific limitations in the expressive power of the underlying language, the logic of the suppositional interpretation is then preserved in the assertibility approach provided that a valid inference is defined as having the sum of the uncertainties of the premises as an upper bound for the uncertainty of the conclusion under any probability assignment. $^{31}$

All this is standard and well received in the literature, especially among authors who_- unlike us-are skeptical either about possible worlds or about the very idea of truth as applied to conditionals. More generally, the logic of the suppositional interpretation largely survives across the divide between accounts based on truth conditions versus assertibility conditions, and this is rightly taken as a sign of the strength of that interpretation. So one might wonder whether something similar can be said about the evidential intepretation. After all, we have relied on the Chrysippus Test in order to convey the idea of support from antecedent to consequent, but support is well known to be amenable to a probabilistic characterization.

As it emerges from Sect. 9, Adams' account is based solely on the Ramsey Test, so it cannot provide a credible reading of the Chrysippus Test. In fact, his account implies that a conditional with antecedent $\alpha$ and consequent $\beta$ can be highly assertible even when $\alpha$ and $\sim \beta$ are compatible in a quite natural sense, and indeed independent, as illustrated by cases such as (11). How can we define assertibility

${ }^{31}$ Adams $(1965,1998)$. 
conditions in probabilistic terms if we think that a conditional is assertible to the extent that the negation of its consequent is incompatible with its antecedent?

Let us consider a conditional $\alpha \triangleright \beta$. How should a plausible probabilistic measure of the incompatibility of $\alpha$ and $\sim \beta$ behave? First, it should be symmetric, as noted in Sect. 2, so that the incompatibility of $\alpha$ with $\sim \beta$ equals the incompatibility of $\sim \beta$ with $\alpha$ for any probability distribution $P$. Second, it should be maximal (that is, 1) in case $P(\alpha \wedge \sim \beta)=0$. Third, it should be minimal (that is, 0 ) in case $\alpha$ and $\sim \beta$ are either probabilistically independent or positively correlated, namely, if $P(\alpha \wedge \sim \beta) \geq P(\alpha) P(\sim \beta)$. The simplest way to meet these constraints, it turns out, is to represent the degree of incompatibility between $\alpha$ and $\sim \beta$ as

$$
1-\frac{P(\alpha \wedge \sim \beta)}{P(\alpha) P(\sim \beta)}
$$

provided that $P(\alpha \wedge \sim \beta) \leq P(\alpha) P(\sim \beta)$, and 0 otherwise. For the limiting cases in which $P(\alpha)=0$ or $P(\beta)=1$, and thus $P(\alpha) P(\sim \beta)=0$, the default option is to say that incompatibility is still maximal (i.e., 1), for then again $P(\alpha \wedge \sim \beta)=0$. If we now follow the idea of the Chrysippus Test and equate the assertibility of $\alpha \triangleright \beta$ to the degree of incompatibility between $\alpha$ and $\sim \beta$, we do recover appropriate connections with current probabilistic analyses of support. ${ }^{32}$ To begin with, $\alpha \triangleright \beta$ turns out to be assertible to some degree given $P$ only if the supposition of $\alpha$ increases the probability of $\beta$ (provided that $\alpha$ has non-null and $\beta$ non-maximal probability), that is, only if $P(\beta \mid \alpha)>P(\beta)$. Moreover, $\alpha \triangleright \beta$ is maximally assertible in case $\alpha$ makes $\beta$ certain, that is, when $P(\beta)<1$ and $P(\beta \mid \alpha)=1$.

Once the assertibility of $\alpha \triangleright \beta$ is characterized in probabilistic terms, one can apply Adams' definition of validity and check the logical principles discussed so far. In an extended investigation along this lines, we have shown that the resulting logic implies exactly the same pattern of results derived from our truth-conditional discussion above. ${ }^{33}$ So the evidential interpretation is similar to the suppositional interpretation in one important respect: its distinctive logical behaviour is robust across alternative frameworks, and can be motivated both in modal terms and in probabilistic terms. This correspondence, we submit, is not accidental, given the underlying idea that the Chrysippus Test plays a key role in our understanding of support.

Acknowledgements We would like to thank Jake Chandler, Paul Egré, Gilberto Gomes, Hitoshi Omori, Carlotta Pavese, Graham Priest, Eric Raidl, Hans Rott, Jan Sprenger, Robert van Rooij, Heinrich Wansing, and one anonymous referee for their helpful comments on previous versions of this paper.

Funding Open access funding provided by Università degli Studi di Torino within the CRUI-CARE Agreement.

Open Access This article is licensed under a Creative Commons Attribution 4.0 International License, which permits use, sharing, adaptation, distribution and reproduction in any medium or format, as long as you give appropriate credit to the original author(s) and the source, provide a link to the Creative

\footnotetext{
32 The resulting measure of assertibility amounts to the probabilistic measure of positive evidential support as partial entailment advocated in Crupi and Tentori (2013, (2014).

33 Crupi and Iacona (2020).
} 
Commons licence, and indicate if changes were made. The images or other third party material in this article are included in the article's Creative Commons licence, unless indicated otherwise in a credit line to the material. If material is not included in the article's Creative Commons licence and your intended use is not permitted by statutory regulation or exceeds the permitted use, you will need to obtain permission directly from the copyright holder. To view a copy of this licence, visit http://creativecommons.org/ licenses/by/4.0/.

\section{References}

Adams, E. W. (1965). The logic of conditionals. Inquiry, 8, 166-197.

Adams, E. W. (1998). A primer of probability logic. Stanford: CSLI Publications.

Angell, R. B. (1962). A propositional logic with subjunctive conditionals. Journal of Symbolic Logic, 27, 327-343.

Bennett, J. (2003). A philosophical guide to conditionals. Oxford: Clarendon Press.

Berto, F., \& Özgün, A. (2020). Indicative conditionals: Probabilities and relevance, unpublished manuscript.

Crupi, V., \& Iacona, A. (2020). Three ways of being non-material, submitted manuscript.

Crupi, V., \& Tentori, K. (2013). Confirmation as partial entailment: A representation theorem in inductive logic. Journal of Applied Logic, 11, 364-372.

Crupi, V., \& Tentori, K. (2014). Measuring information and confirmation. Studies in the History and Philosophy of Science, 47, 81-90.

Dancygier, B. (1998). Conditionals and predictions: Time, knowledge and causation in conditional constructions. Cambridge: Cambridge University Press.

Dancygier, B., \& Sweetser, E. (2005). Mental spaces in grammar: Conditional constructions. Cambridge: Cambridge University Press.

Declerck, R., \& Reed, S. (2001). Conditionals: A comprehensive empirical analysis. Berlin: De Gruyter.

Douven, I. (2008). The evidential support theory of conditionals. Synthese, 164, 19-44.

Douven, I. (2016). The epistemology of conditionals. Cambridge: Cambridge University Press.

Edgington, D. (2001). Indicative conditionals. In E.N. Zalta (ed.), Stanford Encyclopedia of Philosophy (Fall 2020 Edition) Stanford University. https://plato.stanford.edu/archives/fall2020/entries/condi tionals/.

Estrada-Gonzáles, L., \& Ramírez-Cámara, E. (2016). A comparison of connexive logics. IFCOLog Journal of Logics and their Applications, 3, 341-355.

Gillies, A. S. (2009). On truth-conditions for if (but not quite only if). Philosophical Review, 118, 325-349.

Gomes, G. (2019). Meaning-preserving contraposition of conditionals. Journal of Pragmatics, 152, 46-60.

Gomes, G. (2020). Concessive conditionals without even if and nonconcessive conditionals with even if. Acta Analytica, 35, 1-21.

Huber, F. (2007). The logic of theory assessment. Journal of Philosophical Logic, 36, 511-538.

Iacona, A. (2018). Indicative conditionals as strict conditionals. Argumenta, 4, 177-192.

Iacona, A. (2020). Strictness and connexivity. Inquiry (published online: 20 Oct 2019).

Kapsner, A. (2019). Humble connexivity. Logic and Logical Philosophy, 28, 513-536.

Kneale, W., \& Kneale, M. (1962). The development of logic. Oxford: Oxford University Press.

Kratzer, A. (2012). Modals and conditionals. Oxford: Oxford University Press.

Lenzen, W. (2019a). A fresh look at the Stoic's debate on the nature of conditionals, unpublished manuscript.

Lenzen, W. (2019b). Leibniz's laws of consistency and the philosophical foundations of connexive logic. Logical and Logical Philosophy, 28, 537-551.

Lewis, D. (1973). Counterfactuals. Oxford: Blackwell.

Lycan, W. G. (2001). Real conditionals. Oxford: Oxford University Press.

Mares, E. (2012). Relevance logic. In E.N. Zalta (ed.), Stanford Encyclopedia of Philosophy (Summer 2020 Edition). Stanford University.https://plato.stanford.edu/archives/sum2020/entries/logic-relev ance/. 
McCall, S. (2012). A history of connexivity. In D. M. Gabbay, et al. (Eds.), Handbook of the history of logic (Vol. 11, pp. 415-449). Amsterdam: Elsevier.

Mill, J. S. (1843). A system of logic, ratiocinative and inductive (8th ed., p. 1882). New York: Harper and Brothers.

Priest, G. (2008). An introduction to non-classical logic: From if to is. Cambridge: Cambridge University Press.

Raidl, E. (2020). Definable conditionals. Topoi (published online: 11 June 2020).

Ramsey, F. (1929). General propositions and causality. In D. H. Mellor (Ed.), Philosophical papers (pp. 145-163). Cambridge: Cambridge University Press.

Rott, H. (1986). Ifs, though, and because. Erkenntnis, 25, 345-370.

Rott, H. (2020). Difference-making conditionals and the relevant Ramsey test. Review of Symbolic Logic (published online: 12 December 2019).

Sanford, D. (2003). If P, then $Q$. London: Routledge.

Spohn, W. (2013). A ranking-theoretical approach to conditionals. Cognitive Science, 37, 1074-1106.

Stalnaker, R. (1991). A theory of conditionals. In F. Jackson (Ed.), Conditionals (pp. 28-45). Oxford: Oxford University Press.

Unterhuber, M. (2016). Beyond System P. Hilbert-style convergence results for conditional logics with a connexive twist. IFCOLog Journal of Logics and their Applications, 3, 377-412.

van Rooij, R., \& Schulz, K. (2019). Conditionals, causality and conditional probability. Journal of Logic, Language and Information, 28, 55-71.

Veltman, F. (1986). Data semantics and the pragmatics of indicative conditionals. In J. S. Reilly, C. A. Ferguson, E. C. Traugott, \& A. T. Meulen (Eds.), On conditionals (pp. 147-167). Cambridge: Cambridge University Press.

Wansing, H. (2020). Connexive logic. In E. Zalta (Ed.), Stanford encyclopedia of philosophy (Spring 2020 Edition). http://plato.stanford.edu/archives/spr2019/entries/logic-connexive/.

Wenmackers, S., Krzyżanowska, K., \& Douven, I. (2013). Inferential conditionals and evidentiality. Journal of Logic, Language and Information, 22, 315-334.

Publisher's Note Springer Nature remains neutral with regard to jurisdictional claims in published maps and institutional affiliations. 